\title{
BMJ Open Global and regional incidence, mortality and disability-adjusted life-years for Epstein-Barr virus-attributable malignancies, 1990-2017
}

\author{
Gulfaraz Khan (D) , ${ }^{1}$ Christina Fitzmaurice, ${ }^{2}$ Mohsen Naghavi, ${ }^{3}$ Luai A Ahmed (D) ${ }^{4}$
}

To cite: Khan G, Fitzmaurice C, Naghavi M, et al. Global and regional incidence, mortality and disability-adjusted lifeyears for Epstein-Barr virusattributable malignancies, 1990-2017. BMJ Open 2020;10:e037505. doi:10.1136/ bmjopen-2020-037505

- Prepublication history and additional material for this paper are available online. To view these files, please visit the journal online (http://dx.doi. org/10.1136/bmjopen-2020037505).

Received 05 February 2020 Revised 15 May 2020 Accepted 14 July 2020

Check for updates

(c) Author(s) (or their employer(s)) 2020. Re-use permitted under CC BY-NC. No commercial re-use. See rights and permissions. Published by BMJ.

${ }^{1}$ Medical Microbiology \& Immunology, United Arab Emirates University College of Medicine and Health Sciences, Al Ain, United Arab Emirates ${ }^{2}$ Department of Medicine, University of Washington, Seattle, Washington, USA ${ }^{3}$ Institute for Health Metrics and Evaluation, University of Washington, Seattle, Washington, USA

${ }^{4}$ Institute of Public Health, United Arab Emirates University College of Medicine and Health Sciences, Al Ain, United Arab Emirates

Correspondence to Dr Gulfaraz Khan; g_khan@uaeu.ac.ae

\section{ABSTRACT}

Objective To determine the global and regional burden of Epstein-Barr virus (EBV)-attributed malignancies.

Design An international comparative study based on the Global Burden of Disease (GBD) Study estimates.

Setting Global population by age, sex, region, demographic index and time.

Methods and outcome measures The burden of EBVattributed Burkitt lymphoma (BL), Hodgkin lymphoma (HL), nasopharyngeal carcinoma (NPC) and gastric carcinoma (GC) was estimated in a two-step process. In the first step, the fraction of each malignancy attributable to EBV was estimated based on published studies; this was then applied to the GBD estimates to determine the global and regional incidence, mortality and disability-adjusted life-years (DALYs) for each malignancy by age, sex, geographical region and social demographic index (SDI) from 1990 to 2017.

Results The combined global incidence of $\mathrm{BL}, \mathrm{HL}$, NPC and GC in 2017 was 1.442 million cases, with over 973000 deaths. An estimated 265000 (18\%) incident cases and 164000 (17\%) deaths were due to the EBVattributed fraction. This is an increase of $36 \%$ in incidence and $19 \%$ in mortality from 1990. In 2017, EBV-attributed malignancies caused 4.604 million DALYs, of which $82 \%$ was due to NPC and GC alone. The incidence of both of these malignancies was higher in high and middle-high SDI regions and peaked in adults aged between 50 and 70 years. All four malignancies were more common in males and the highest burden was observed in East Asia. Conclusions This study provides comprehensive estimates of the burden of EBV-attributed BL, HL, NPC and GC. The overall burden of EBV-related malignancies is likely to be higher since EBV is aetiologically linked to several other malignancies not included in this analysis. Increasing global population and life expectancy is expected to further raise this burden in the future. The urgency for developing an effective vaccine to prevent these malignancies cannot be overstated.

\section{INTRODUCTION}

Cancer is one of the leading causes of death worldwide. The latest estimates indicate that in 2017 , there were nearly 17 million new cases and over 9 million deaths worldwide. ${ }^{1}$ Alarmingly, the overall burden due to cancer is on

\section{Strengths and limitations of this study}

- This study examined the burden of Epstein-Barr virus (EBV)-attributed malignancies using the most up-to-date and reliable data from the Global Burden of Disease (GBD) Study.

- This is the first study of its kind to quantitate the global and regional incidence, mortality and disability-adjusted life-years of EBV-attributed malignancies by age, sex, geographical region and social demographic index from 1990 to 2017.

- Although EBV is linked to a number of malignancies, this study assessed only four EBV-associated malignancies.

- The GBD estimates, although the most comprehensive and most refined, have their own drawbacks and limitations.

the rise, primarily due to population growth and increasing life expectancy. ${ }^{1}$ Cancer is a complex and multifactorial disease and strategies to reduce its burden will require not only basic research, but also a global action plan targeting early detection, control and prevention. One fundamental aspect of prevention is to understand the causes of cancers. It is now well-established that infectious agents, either on their own or in combination with genetic and environmental factors, play a role in the pathogenesis of approximately $15 \%-20 \%$ of all human malignancies. ${ }^{2-4}$ Most of these malignancies are linked to only a handful of infectious agents. ${ }^{45}$ One such agent is Epstein-Barr virus (EBV).

EBV is a very common virus asymptomatically infecting over $90 \%$ of the population. ${ }^{6}$ In most cases, the infection is acquired early in childhood, often before the age of 5 years. ${ }^{7}$ Once infected, the virus persists in B cells for life. ${ }^{8}$ Depending on the pattern of EBV gene expression in the infected cells, four latency programmes, referred to as latency 0 to 3 , have been recognised. Different latency programmes are associated with different pathologies. ${ }^{9}$ 
Moreover, the fact that $\mathrm{EBV}$ is very common in the general population, and yet only a very small fraction of infected individuals develop EBV-associated pathologies, indicates that other risk factors such as immune deficiencies, genetic predisposition and environmental factors are also essential in the development of these pathologies. ${ }^{10-12}$ Thus, to establish a causal link, it is necessary to directly demonstrate the virus in the affected tissues. With the advancement in technology and detection methods, the virus has now been unequivocally demonstrated in the tumour cells of several different malignancies. ${ }^{9} \mathrm{EBV}$ is now firmly linked to the development of Burkitt lymphoma (BL), nasopharyngeal carcinoma (NPC), Hodgkin lymphoma (HL) and gastric carcinomas (GCs). ${ }^{9}$ Additionally, EBV is also clearly implicated in the pathogenesis of several other malignancies, including lymphomas arising in immunocompromised individuals, such as allograft recipients, patients with AIDS and individuals with congenital immunodeficiencies. ${ }^{13} 14$

Although EBV was the first virus identified to be aetiologically associated with human malignancies, no effective antiviral drug or approved vaccine is available for its elimination or prevention. An accurate estimate of the burden of EBVattributed disorders is unknown. The purpose of this study was to partially fill this gap by providing estimates of EBVattributed BL, HL, NPC and GC, using the Global Burden of Disease (GBD 2017) estimates.

\section{METHODS}

\section{Patients and public involvement}

This study was based on an open-access database available from the website of the Institute of Health Metrics and Evaluation, University of Washington. The database has no identifiable information on the patients. Patients or the public were not involved in the design, or conduct, or reporting, or dissemination plans of our research.

\section{Definition and prevalence of EBV-attributable cases of BL, HL, NPC and GC}

For the purpose of this study, we defined EBV-attributable cancers as those in which viral nucleic acid, and/or viral proteins can be demonstrated directly in the malignant cells (table 1). This has consistently and unequivocally been confirmed for BL, HL, NPC and GC by numerous studies (table 1). However, not all of the cases of these malignancies are EBV attributable. Moreover, the EBV-attributable fraction appears to vary with age, gender and geographical region. Taking these variables into account, we first estimated the fraction of EBV-attributable malignancies based on published studies as described in our previous study. ${ }^{15}$ Table 1 summarises the outcome of this analysis.

\section{Estimation of the incidence, mortality and DALYs for BL, HL, NPC and GC}

Estimates of incidence, mortality and disability-adjusted life-years (DALYs) for HL, NPC and GC were obtained from the GBD 2017 study. GBD methods are described extensively elsewhere. ${ }^{11617}$ Briefly, estimates are based on multiple data sources, including vital registration systems, cancer registries and verbal autopsy data. ${ }^{1617}$ A range of statistical models were used to derive the final estimates. Since each GBD study re-estimates the entire data sets annually, the results presented here are the most refined and up-to-date.

Datasets of age and sex-specific estimates of incidence, mortality and DALYs for 21 global regions from 1990 to 2017 were directly available from the GBD results database for HL, NPC and GC (http:/ /ghdx.healthdata.org/ gbd-results-tool, downloaded on 24 March 2019). For $\mathrm{BL}$, no direct estimates were available. The GBD study includes BL under the broader category of non-Hodgkin lymphomas (NHLs). Based on previous studies, ${ }^{2}{ }^{15}$ we estimated the percentage of BL cases within the NHLs category in the age group $0-14$ years to be $90.5 \%, 33.3 \%$ and $15.2 \%$ for regions where $\mathrm{BL}$ is endemic, intermediate or sporadic, respectively (table 1). For age group 15-80+, irrespective of geographical region, the percentage of $\mathrm{BL}$ in HIV-negative adults was conservatively estimated to be $2 \%$ of all NHLs cases. ${ }^{18}$ BL is approximately 3-4 times more common in males compared with females. ${ }^{19-21}$ In this study, we used male:female ratio of 3:1 in calculating the prevalence of BL. Thus, for BL, we first estimated the incidence, mortality and DALYs by age, sex and geographical region, before calculating the fractions attributable to EBV.

\section{Estimation of the incidence, mortality and DALYs of EBV-} attributed fraction of BL, HL, NPC and GC

The EBV-attributable proportion of BL, HL, NPC and GC estimated from published studies (table 1) was applied to the GBD 2017 estimates. For example, for BL in East SubSaharan Africa, GBD 2017 estimates show 1526 incident cases of NHLs in the age group 1-4 years. In this region, $90.5 \%$ of NHLs cases have been estimated to be BL in this age group, ${ }^{2}$ with a male predominance of 3:1. ${ }^{19} 2022$ Based on this, $1526 \times 0.905 \times 0.75$ gives an estimate of the incidence of BL in males in the 1-4year age group in East Sub-Saharan Africa in 2017 to be 1036. Since 95\% of BL cases in this age group and in this region are EBV associated, ${ }^{23}$ the incidence of EBV-attributed BL cases was estimated to be 984 cases $(1036 \times 0.95)$. Using this approach, we calculated the incidence, mortality and DALYs for each of the four EBV-associated malignancies in males and females in 23 different age groups and 21 different geographical regions from 1990 to 2017.

\section{Estimation of the burden of EBV-attributed fraction of $B L, H L$, NPC and GC by SDI}

To assess the influence of demographic development on the burden of EBV-attributable malignancies, we used each country's social demographic index (SDI) to estimate EBV-attributed fraction of BL, HL, NPC and GC. SDI is a summary measure of the lag distributed income per capita, educational attainment and fertility rate and it is regarded as a good indicator of a country's sociodemographic development. ${ }^{1}$ We assessed the burden of 
Table 1 Characteristics and prevalence of EBV-attributable cases of BL, HL, NPC and GC

\begin{tabular}{|c|c|c|c|c|c|}
\hline Malignancy & $\begin{array}{l}\text { Comment on age, gender, } \\
\text { regional variations }\end{array}$ & $\begin{array}{l}\text { Prevalence } \\
\text { of EBV in } \\
\text { cases (\%) }\end{array}$ & $\begin{array}{l}\text { Cellular origin } \\
\text { of malignant } \\
\text { cells }\end{array}$ & $\begin{array}{l}\text { Pattern of EBV } \\
\text { gene expression } \\
\text { in malignant cells }\end{array}$ & References \\
\hline \multicolumn{6}{|l|}{$\mathrm{BL}$} \\
\hline $\begin{array}{l}\text { Endemic regions } \\
\text { (M:F ratio } 3: 1)\end{array}$ & $\begin{array}{l}\text { Sub-Saharan Africa have highest } \\
\text { risk }\end{array}$ & 95 & B cells & $\begin{array}{l}\text { Type I latency } \\
\text { (EBERs, EBNA1) }\end{array}$ & $19-222526$ \\
\hline $\begin{array}{l}\text { Intermediate regions } \\
(\mathrm{M}: \mathrm{F} \text { ratio } 3: 1)\end{array}$ & $\begin{array}{l}\text { North Africa and Middle East, } \\
\text { Latin America, have intermediate } \\
\text { risk }\end{array}$ & 50 & & & \\
\hline $\begin{array}{l}\text { Non-endemic } \\
\text { regions (M:F ratio } \\
3: 1)\end{array}$ & All other regions have low risk & 20 & & & \\
\hline \multicolumn{6}{|l|}{ HL } \\
\hline Adults $>55$ years & $\begin{array}{l}\text { Age group } 55+\text { years have } \\
\text { medium/high risk }\end{array}$ & 55 & & & \\
\hline \multicolumn{6}{|l|}{ NPC } \\
\hline $\begin{array}{l}\text { High/intermediate } \\
\text { incident regions }\end{array}$ & $\begin{array}{l}\text { East Asia, South Asia, South East } \\
\text { Asia, North Africa and Middle East }\end{array}$ & 100 & Epithelial cells & $\begin{array}{l}\text { Type Il latency } \\
\text { (EBERs, EBNA1, } \\
\text { LMP1) }\end{array}$ & 2323 \\
\hline $\begin{array}{l}\text { Low incident } \\
\text { regions }\end{array}$ & All other regions & 80 & & & \\
\hline \multicolumn{6}{|l|}{ GC } \\
\hline
\end{tabular}

Based on published studies, we estimated the proportion of BL, HL, NPC and GC that are attributable to EBV, taking into consideration any established variations that have been reported in different age, sex and ethnic groups. The cellular origin of each malignancy and the pattern of EBV gene expression is also indicated.

BL, Burkitt lymphoma; EBERs, Epstein-Barr encoded RNA; EBNA, Epstein-Barr nuclear antigen; EBV, Epstein-Barr virus; GC, gastric carcinoma; HL, Hodgkin lymphoma; LMP, latent membrane protein; NPC, nasopharyngeal carcinoma.

EBV-attributed malignancies by five SDI categories: low, low-middle, middle, middle-high and high. ${ }^{1}$

\section{RESULTS}

\section{Global burden of EBV-attributed malignancies}

In 2017, there were 1.442 million incident cases and 973000 deaths from BL, HL, NPC and GC, contributing to 22.958 million DALYs (table 2). The overall global burden of EBV-attributed fraction of these four malignancies contributed to over $265000(18 \%)$ of the incident cases, $164000(17 \%)$ of deaths and 4.6 million (20\%) of DALYs (table 2 and online supplementary figure 1). The individual contribution of each of these four malignancies to the overall burden of EBV-attributed fractions varied considerably. NPC and GC together accounted for over 218000 (82\%) incident cases, 146000 (89\%) deaths and 3.8 million (82\%) DALYs (table 2). Over the period of 27 years (1990-2017), the burden of mortality from these EBV-attributed malignancies increased by $19 \%$.

\section{EBV-attributed malignancies by sex and age}

The incidence and mortality of all four malignancies (BL, HL, NPC and GC) were higher in males than in females in all world regions. The EBV-attributed fraction of these malignancies was also higher in males compared with females (figure 1). The combined incidence of EBVattributed BL, HL, NPC and GC in 2017 was 196000 in males and 69000 in females (2.8:1.0, table 2). Incidence and mortality of EBV-attributed malignancies also varied with age (see online supplementary figures 2,3 ). BL was primarily seen in children, peaking in the 5-10year age group (see online supplementary figure 2A). By contrast, NPC and GC occurred in adults, peaking in the 45-60 (see online supplementary figure 2C) and 65-80 (see online supplementary figure 2D) age group, respectively. 

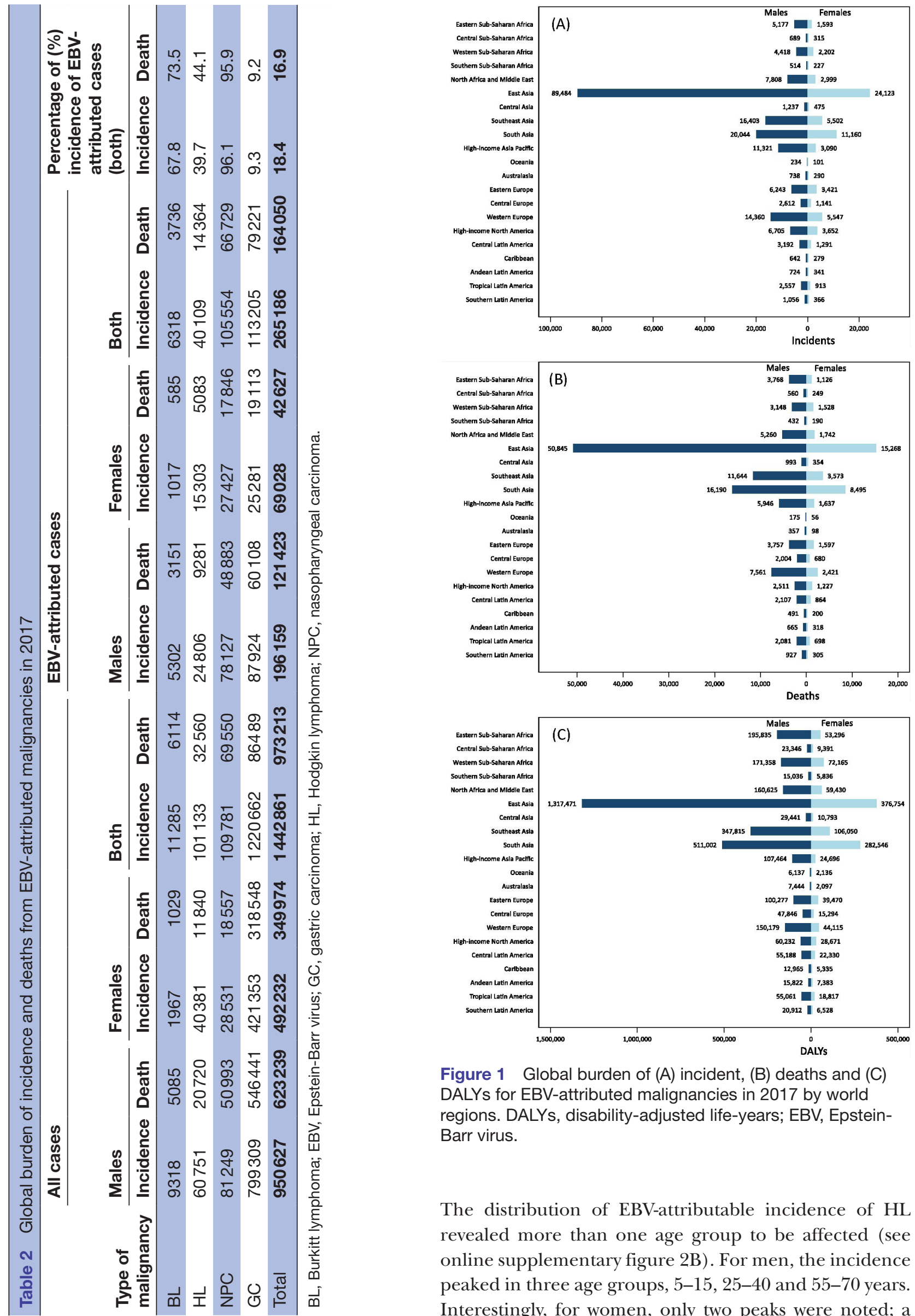

Figure 1 Global burden of (A) incident, (B) deaths and (C) DALYs for EBV-attributed malignancies in 2017 by world regions. DALYs, disability-adjusted life-years; EBV, EpsteinBarr virus.

The distribution of EBV-attributable incidence of HL revealed more than one age group to be affected (see online supplementary figure $2 \mathrm{~B}$ ). For men, the incidence peaked in three age groups, $5-15,25-40$ and $55-70$ years. Interestingly, for women, only two peaks were noted; a 
large peak in the 25-40year age group and a smaller peak in the 55-70year group.

\section{EBV-attributed malignancies by region and time}

There was considerable regional variation in the burden of EBV-attributed BL, HL, NPC and GC (see online supplementary figures 4-6). This ranged from less than 1000 incident cases in Southern Sub-Saharan Africa to more than 100000 cases in East Asia (see online supplementary figure 1). In fact, $43 \%$ of all global incident cases of these four malignancies and $40 \%$ of all deaths were in East Asia (figure 1). This high burden is primarily due to the high incidence of NPC and GC in East Asia (see online supplementary figures 6,7), particularly in China (see online supplementary figure 1 ).

The combined incidence of the four EBV-attributed malignancies has increased from 195000 in 1990 to 265000 in 2017. This increase is particularly evident for males (figure 2A). For females, the increase has been either moderate, or in the case of NPC, actually decreased slightly (277000 in 1990 to 274000 in 2017). In terms of the burden of deaths, absolute numbers declined only for HL (figure 2B). Since the global population has increased over the same timeframe, the rate of death per 100000 for HL has decreased significantly from 0.67 in 1990 to 0.43 in 2017.

\section{EBV-attributed malignancies by SDI}

Since a country's socioeconomic development is an important driver of the burden of disease, we assessed the impact of SDI on the burden of EBV-attributed malignancies. Countries were grouped into five categories, low, low-middle, middle, middle-high and high SDI. As expected, there was considerable heterogeneity in both incidence and mortality by SDI status (figure 3 ). For EBVattributed BL, low and low-middle SDI regions had the highest burden of incidence and deaths, while for EBVattributed HL, incidence appeared to directly correlate with the SDI index; the highest burden was observed in the high SDI region (figure 3B). The burden of deaths from EBV-attributed HL on the other hand, did not follow the pattern seen for incident cases. The burden of deaths was greater in low and low-middle SDI regions, possibly reflecting less resources for treating HL in these regions compared with the affluent high SDI countries (figure 3B). The burden of DALYs for EBV-attributed cases also varied by SDI; low and low-middle countries had the highest burden of BL and HL, but for NPC and GC, the highest burden was observed in middle and middlehigh countries (see online supplementary figure 8).

\section{DISCUSSION}

Improvements in life expectancy and population growth has led to an increase in the global burden of cancer, which now ranks second after cardiovascular diseases. ${ }^{1}$ To address this growing global health problem, a multipronged approach is needed. It is essential not only to
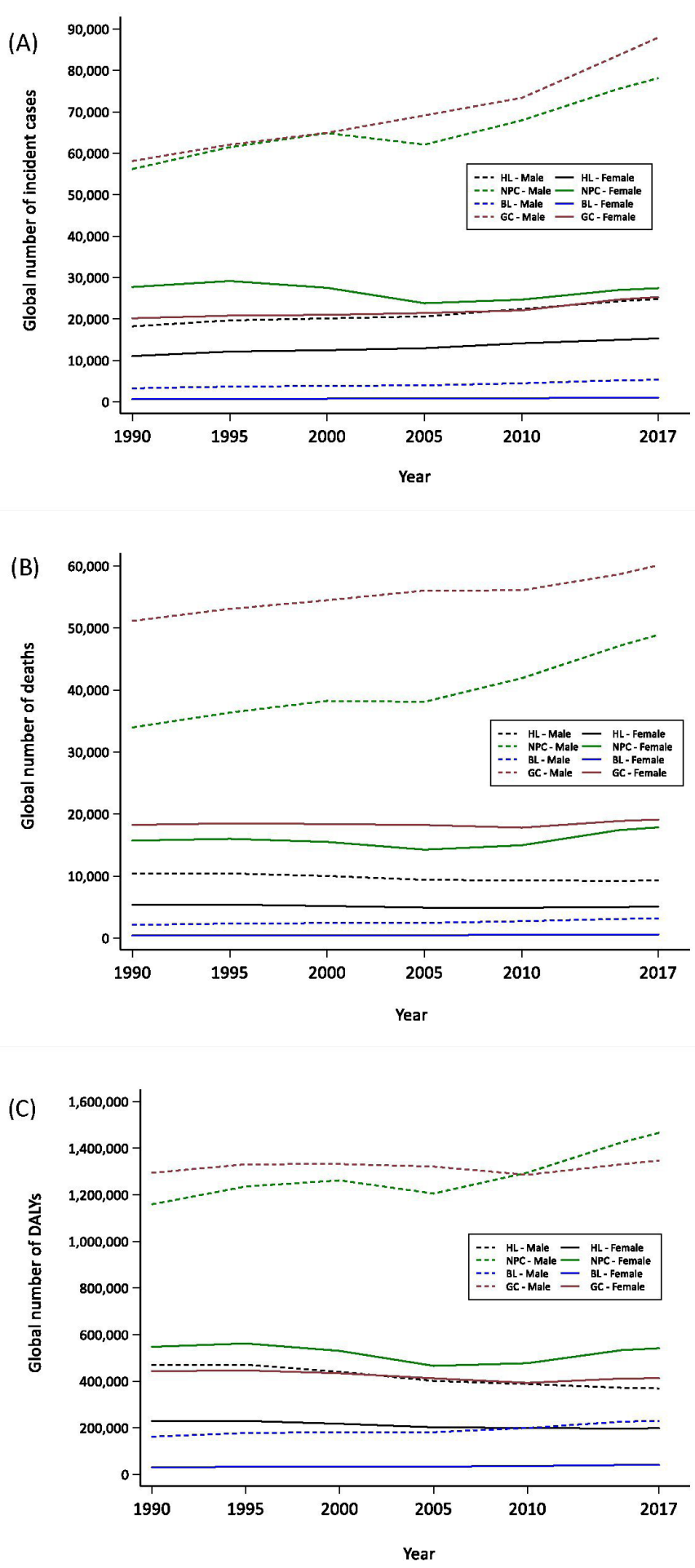

Figure 2 Global burden of incident (A), deaths (B) and DALYs (C) for EBV-attributed malignancies by gender from 1990 to 2017. BL, Burkitt lymphoma; DALYs, disabilityadjusted life-years; EBV, Epstein-Barr virus; GC, gastric carcinoma; HL, Hodgkin lymphoma; NPC, nasopharyngeal carcinoma.

find better therapies for cancer, but importantly, to prevent cancer from occurring in the first place. Thus, understanding the causes and risk factors involved in the development of cancer is of central importance. In this first of a kind study, we provide detailed estimates of the 
(A)

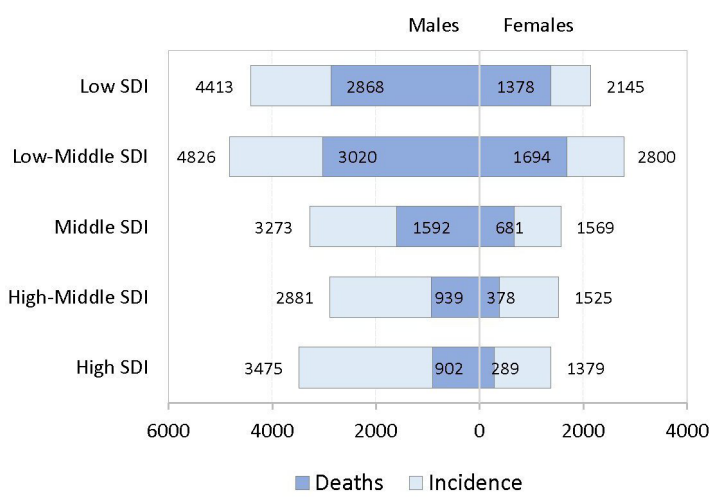

(C)

EBV-attributed cases of NPC

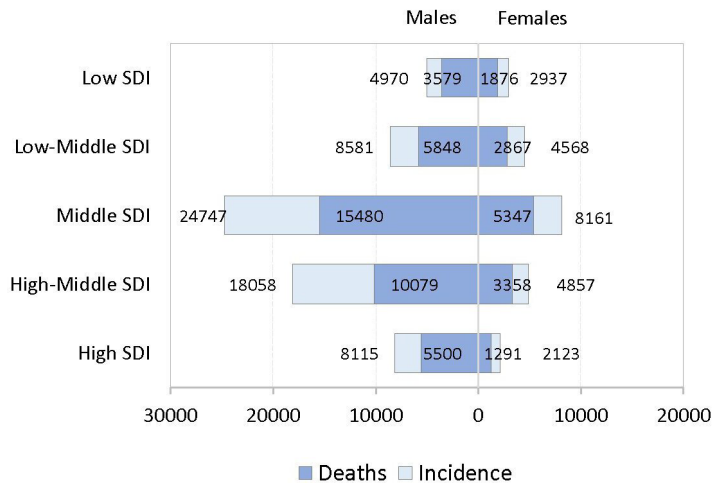

(B)

EBV-attributed cases of $\mathrm{HL}$

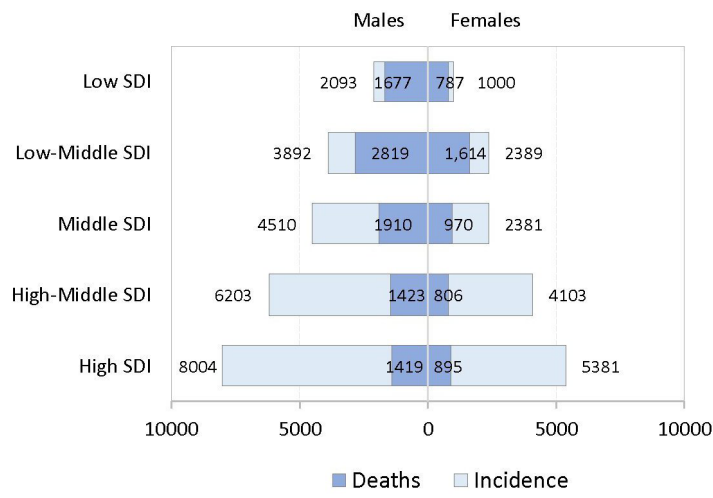

(D)

EBV-attributed cases of GC

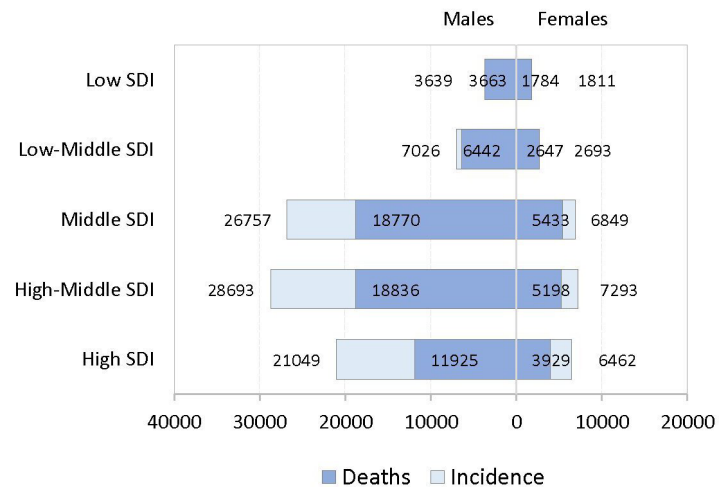

Figure 3 Global burden of incident and deaths of EBV-attributed (A) BL, (B) HL, (C) NPC and (D) GC in 2017 by SDI world regions. BL, Burkitt lymphoma; EBV, Epstein-Barr virus; GC, gastric carcinoma; HL, Hodgkin lymphoma; NPC, nasopharyngeal carcinoma; SDI, social demographic index.

global and regional incidence, mortality and DALYs of EBV-attributed malignancies by age, sex, geographical region and SDI from 1990 to 2017.

Our analysis revealed that in 2017, BL, HL, NPC and GC accounted for 1.44 million incident cases and almost 1 million deaths. Of these, just over 265000 incident cases and 164000 deaths were attributed to EBV infection. This is an increase of $19 \%$ and $36 \%$, respectively from 1990 . Since the global prevalence and pattern of EBV infection has not changed, the drivers of the increase in burden of EBV-attributed cancers appear to be due to an increase in the life expectancy, population growth and changing age structure. ${ }^{1}$ The contribution of these drivers varies with socioeconomic development. While, population growth is a major driver of the increased burden in low SDI regions, increased life expectancy appears to be more important in middle-high and high SDI regions. ${ }^{1}$ As previously reported, all four malignancies were more common in males. ${ }^{1}$ The reasons for the male preponderance is not known, but genetics and male life-style risk factors are likely to be important contributors. ${ }^{23} 24$

The fraction of cases attributed to EBV also varied significantly depending on the type of malignancy. While more than $95 \%$ of NPC cases were attributed to EBV, for GC, this fraction was $<10 \%$. In spite of this low attributed fraction, GC was still the leading cause of
EBV-attributed cancer burden, accounting for $43 \%$ of all incident cases and nearly $50 \%$ of all deaths in 2017 . This burden is due to the fact that GC is among the top six most frequently diagnosed cancer globally, and the most common cancer in some East Asian countries. ${ }^{1}$ Although the absolute number of EBV-attributed GC incident cases has increased from 78000 in 1990 to over 113000 in 2017, the age-standardised incidence rate has actually declined globally. GC peaks in late adulthood (above 65 years) and the absolute increase in incidence of GC could be explained by the increase in life expectancy and change in population age structure. East Asia also had by far the highest incidence of NPC. In fact, approximately $50 \%$ of the global number of EBV-attributed cases of GC and NPC occurred in East Asia. The reasons for the high prevalence of these two malignancies in this region is not clear. It is believed that a combination of genetic and environmental risk factors are involved. Early infection with EBV and/or Helicobacter pylori, both of which are common in the region are important risk factors, as is diet, high salt intake, smoking and life-style factors. ${ }^{2324}$ A change in exposure to these risk factors has been reported to reduce the incidence rates, as shown in studies on descendants of migrants from high to low incidence regions. ${ }^{23}$

In contrast to NPC and GC, the epidemiology of BL and HL is very different. BL is a childhood malignancy 
most prevalent in Eastern and Western Sub-Saharan Africa. Males are more predominantly affected. ${ }^{19-22} 2526$ Three risk factors have been shown to be involved in the development of BL; EBV infection, malaria and genetic translocation involving the $c-m y c$ oncogene. ${ }^{9}$ However, the level of contribution of each of these risk factors and how they interact to promote the development of $\mathrm{BL}$ is unknown. As for HL, this study shows that around $40 \%$ of all cases worldwide are EBV-attributed and this fraction varies not only by gender and age, but also by geographical region. ${ }^{27}$ These variations suggest that other risk factors, in addition to EBV are involved in the pathogenesis of HL. Studies have demonstrated that infectious mononucleosis, a self-limiting lymphoproliferative condition caused by primary EBV infection, is associated with a significantly increased risk of developing HL. ${ }^{9}$

\section{LIMITATIONS}

The analysis presented in this report is to our knowledge the most comprehensive and up-to-date assessment of the magnitude and distribution of EBV-attributed malignancies. However, the accuracy of the results rely on a number of predictions and assumptions. First, our estimates of the burden of EBV-attributed BL, HL, NPC and GC were calculated based on the GBD 2017 estimates on incidence, mortality and DALYs of these malignancies. The GBD study, although the most comprehensive and most refined, nevertheless has its own drawbacks and limitations. ${ }^{117}$ Second, GBD groups BL as part of the larger category of NHLs. In this study, when calculating the incidence, mortality and DALYs for BL, we assumed that these measures were proportionally the same for all the lymphomas in the NHLs group. In reality this is not quite true. ${ }^{28}$ In future GDB studies, we aim to include BL as a separate entity, which will provide more accurate estimates. Third, although age, gender and regional variations in EBV-attributable fractions of these malignancies were taken into consideration, we assumed that the EBVattributable fraction was the same for incidence, mortality and DALYs. Fourth, it is currently unclear if EBV-attributed malignancies have a better or worse prognosis. Thus, in this study we assumed that the mortality and DALYs was the same for EBV-associated and non-associated cancers. Similarly, it was assumed that the mortality from EBVattributed malignancies was the same in both males and females. In spite of these limitations, this is the only study of its kind to provide a detailed picture of incidence, mortality and DALYs of EBV-attributed malignancies by age, sex, geographical region and SDI.

\section{CONCLUSION}

Our study shows that EBV-attributed malignancies account for a sizeable fraction of the global burden of cancer. Increasing global population and life expectancy will further increase this burden. It is possible to prevent or at least significantly reduce this burden if an effective vaccine was available. ${ }^{29}$ Future efforts should be aimed at accelerating and expanding vaccine developments. ${ }^{30}$

Contributors GK conceptualised the study and prepared the first draft. LAA and GK did the analysis and prepared the figures and tables. GK, LAA, CF and MN contributed to data interpretation and drafting of the manuscript. All coauthors approved the final draft of the manuscript.

Funding The authors have not declared a specific grant for this research from any funding agency in the public, commercial or not-for-profit sectors.

Map disclaimer The depiction of boundaries on this map does not imply the expression of any opinion whatsoever on the part of BMJ (or any member of its group) concerning the legal status of any country, territory, jurisdiction or area or of its authorities. This map is provided without any warranty of any kind, either express or implied.

Competing interests None declared.

Patient consent for publication Not required.

Provenance and peer review Not commissioned; externally peer reviewed.

Data availability statement Data are available in a public, open access repository. All data relevant to the study are included in the article or uploaded as supplementary information. Data are publicly available from the Global Burden of Disease Study (http://ghdx.healthdata.org/gbd-results-tool).

Open access This is an open access article distributed in accordance with the Creative Commons Attribution Non Commercial (CC BY-NC 4.0) license, which permits others to distribute, remix, adapt, build upon this work non-commercially, and license their derivative works on different terms, provided the original work is properly cited, appropriate credit is given, any changes made indicated, and the use is non-commercial. See: http://creativecommons.org/licenses/by-nc/4.0/.

ORCID iDs

Gulfaraz Khan http://orcid.org/0000-0001-6836-1783

Luai A Ahmed http://orcid.org/0000-0001-5292-8212

\section{REFERENCES}

1 Global Burden of Disease Cancer Collaboration, Fitzmaurice C, Abate D, et al. Global, regional, and National cancer incidence, mortality, years of life lost, years lived with disability, and disabilityadjusted life-years for 29 cancer groups, 1990 to 2017: a systematic analysis for the global burden of disease study. JAMA Oncol 2019;4:1749-68.

2 Parkin DM. The global health burden of infection-associated cancers in the year 2002. Int J Cancer 2006;118:3030-44.

3 de Martel C, Ferlay J, Franceschi S, et al. Global burden of cancers attributable to infections in 2008: a review and synthetic analysis. Lancet Oncol 2012;13:607-15.

4 Plummer M, de Martel C, Vignat J, et al. Global burden of cancers attributable to infections in 2012: a synthetic analysis. Lancet Glob Health 2016;4:e609-16.

5 Pagano JS, Blaser M, Buendia M-A, et al. Infectious agents and cancer: criteria for a causal relation. Semin Cancer Biol 2004;14:453-71.

6 Young LS, Yap LF, Murray PG. Epstein-Barr virus: more than 50 years old and still providing surprises. Nat Rev Cancer 2017;12:789-802.

7 Fleisher G, Henle W, Henle G, et al. Primary infection with EpsteinBarr virus in infants in the United States: clinical and serologic observations. J Infect Dis 1979;139:553-8.

8 Khan G, Miyashita EM, Yang B, et al. Is EBV persistence in vivo a model for B cell homeostasis? Immunity 1996;5:173-9.

9 Longnecker R, Kieff E, Cohen JI. Epstein-barr virus. In: Knipe DM, Howley PM, eds. Fields virology. Philadelphia: Lippincott Williams \& Wilkins, 2013: 1898-959.

10 Cohen Jl. Epstein-barr virus infection. N Engl J Med Overseas Ed 2000;343:481-92.

11 Rigaud S, Fondanèche M-C, Lambert N, et al. XIAP deficiency in humans causes an X-linked lymphoproliferative syndrome. Nature 2006;444:110-4.

12 Münz C, Moormann A. Immune escape by epstein-barr virus associated malignancies. Semin Cancer Biol 2008;18:381-7.

13 Carbone A, Gloghini A, Dotti G. EBV-associated lymphoproliferative disorders: classification and treatment. Oncologist 2008;13:577-85.

14 Coffey AJ, Brooksbank RA, Brandau O, et al. Host response to $\mathrm{EBV}$ infection in $\mathrm{X}$-linked lymphoproliferative disease results 
from mutations in an SH2-domain encoding gene. Nat Genet 1998;20:129-35

15 Khan G, Hashim MJ. Global burden of deaths from epstein-barr virus attributable malignancies 1990-2010. Infect Agent Cancer 2014;9:38

16 Dicker D, Nyguyen D, Abate KH, et al. Global, regional, and national age-sex-specific mortality and life expectancy, 1950-2017: a systematic analysis for the global burden of disease study 2017. Lancet 2018;392:1684-735.

17 Roth GA, Abate D, Abate KH, et al. Global, regional, and national age-sex-specific mortality for 282 causes of death in 195 countries and territories, 1980-2017: a systematic analysis for the global burden of disease study 2017. Lancet 2018;392:1736-88.

18 Spina M, Tirelli U, Zagonel V, et al. Burkitt's lymphoma in adults with and without human immunodeficiency virus infection: a single-institution clinicopathologic study of 75 patients. Cancer 1998;82:766-74.

19 Philip T. Burkitt's lymphoma in Europe. IARC Sci Publ 1985;60:107-18.

20 Boerma EG, van Imhoff GW, Appel IM, et al. Gender and age-related differences in Burkitt lymphoma-epidemiological and clinical data from The Netherlands. Eur J Cancer 2004;40:2781-7.

21 Magrath IT. African Burkitt's lymphoma. history, biology, clinical features, and treatment. Am J Pediatr Hematol Oncol 1991;13:222-46.

22 Hsu JL, Glaser SL. Epstein-barr virus-associated malignancies: epidemiologic patterns and etiologic implications. Crit Rev Oncol Hematol 2000;34:27-53.

23 Chang ET, Adami H-O. The enigmatic epidemiology of nasopharyngeal carcinoma. Cancer Epidemiol Biomarkers Prev 2006;15:1765-77.

24 Joossens JV, Hill MJ, Elliott P, et al. Dietary salt, nitrate and stomach cancer mortality in 24 countries. European cancer prevention (ECP) and the INTERSALT cooperative Research Group. Int J Epidemiol 1996;25:494-504.

25 Stefan DC, Lutchman R. Burkitt lymphoma: epidemiological features and survival in a South African centre. Infect Agent Cancer 2014;9:19.
26 Queiroga EM, Gualco G, Weiss LM, et al. Burkitt lymphoma in Brazil is characterized by geographically distinct clinicopathologic features. Am J Clin Pathol 2008;130:946-56.

27 Glaser SL, Lin RJ, Stewart SL, et al. Epstein-Barr virus-associated Hodgkin's disease: epidemiologic characteristics in international data. Int J Cancer 1997;70:375-82.

28 Bowzyk Al-Naeeb A, Ajithkumar T, Behan S, et al. Non-Hodgkin lymphoma. BMJ 2018;362:k3204.

29 Cohen JI, Fauci AS, Varmus H, et al. Epstein-Barr virus: an important vaccine target for cancer prevention. Sci Trans/ Med 2011;3:107fs7.

30 Boyle M. A vaccine to kiss EBV goodbye. Sci Transl Med 2019;11:eaax1729.

31 Jarrett AF, Armstrong AA, Alexander E. Epidemiology of EBV and Hodgkin's lymphoma. Ann Oncol 1996;7:S5-10.

32 Murray PG, Billingham LJ, Hassan $\mathrm{HT}$, et al. Effect of epsteinbarr virus infection on response to chemotherapy and survival in Hodgkin's disease. Blood 1999;94:442-7.

33 Stark GL, Wood KM, Jack F, et al. Hodgkin's disease in the elderly: a population-based study. Br J Haematol 2002;119:432-40.

34 Herling M, Rassidakis GZ, Medeiros LJ, et al. Expression of epsteinbarr virus latent membrane protein-1 in Hodgkin and Reed-Sternberg cells of classical Hodgkin's lymphoma: associations with presenting features, serum interleukin 10 levels, and clinical outcome. Clin Cancer Res 2003;9:2114-20.

35 Jarrett RF, Stark GL, White J, et al. Impact of tumor epstein-barr virus status on presenting features and outcome in age-defined subgroups of patients with classic Hodgkin lymphoma: a population-based study. Blood 2005;106:2444-51.

36 Glaser SL, Gulley ML, Clarke CA, et al. Racial/ethnic variation in EBVpositive classical Hodgkin lymphoma in California populations. Int $J$ Cancer 2008;123:1499-507.

37 Murphy G, Pfeiffer R, Camargo MC, et al. Meta-analysis shows that prevalence of epstein-barr virus-positive gastric cancer differs based on sex and anatomic location. Gastroenterology 2009;137:824-33.

38 Lee J-H, Kim S-H, Han S-H, et al. Clinicopathological and molecular characteristics of epstein-barr virus-associated gastric carcinoma: a meta-analysis. J Gastroenterol Hepatol 2009;24:354-65. 